University of Nebraska - Lincoln

DigitalCommons@University of Nebraska - Lincoln

Faculty Publications, Department of Psychology

Psychology, Department of

2011

\title{
Treatment effects for common outcomes of child sexual abuse: A current meta-analysis
}

\author{
Emily V. Trask \\ University of Nebraska-Lincoln, evtrask@ucsd.edu \\ Kate Walsh \\ University of Nebraska-Lincoln, katelynwalsh@hotmail.com \\ David DiLillo \\ University of Nebraska-Lincoln, ddilillo@unl.edu
}

Follow this and additional works at: https://digitalcommons.unl.edu/psychfacpub

Part of the Psychiatry and Psychology Commons

Trask, Emily V.; Walsh, Kate; and DiLillo, David, "Treatment effects for common outcomes of child sexual abuse: A current meta-analysis" (2011). Faculty Publications, Department of Psychology. 573.

https://digitalcommons.unl.edu/psychfacpub/573

This Article is brought to you for free and open access by the Psychology, Department of at DigitalCommons@University of Nebraska - Lincoln. It has been accepted for inclusion in Faculty Publications, Department of Psychology by an authorized administrator of DigitalCommons@University of Nebraska - Lincoln. 
Published in Aggression and Violent Behavior 16 (2011), pp. 6-19; doi:10.1016/j.avb.2010.10.001

Copyright $\odot 2010$ Elsevier Ltd. Used by permission.

Submitted July 1, 2010; revised and accepted October 20, 2010; published online October 26, 2010.

\title{
Treatment effects for common outcomes of child sexual abuse: A current meta-analysis
}

\author{
Emily V. Trask, Kate Walsh, and David DiLillo \\ University of Nebraska-Lincoln, 238 Burnett Hall, Lincoln, NE 68588-0308, USA \\ Corresponding author - E. V. Trask, Child and Adolescent Services Research Center/University of California, San Diego, 3665 Kearney Villa Road, \\ Suite 200, San Diego, CA 92123, USA; tel 858 966-7703 ×3618, fax 858 966-7704, email evtrask@ucsd.edu
}

\begin{abstract}
The present meta-analysis examined the effects of psychosocial treatments at reducing deleterious outcomes of sexual abuse. The meta-analysis included a total of 35 published and unpublished studies written in English, focusing on youth under the age of 18, and evaluating the effectiveness of treatments for the most common negative outcomes of sexual abuse: PTSD symptoms, externalizing problems, and internalizing problems. Results revealed medium effect sizes for PTSD symptoms, externalizing problems, and internalizing problems following treatment for sexual abuse. This study also examined the potential moderating effects of treatment (e.g., modality, duration, and inclusion of caregiver) and participant (e.g., age, gender, and ethnicity) characteristics. Results indicated that longer interventions were associated with greater treatment gains while group and individual treatments were equally effective. These findings shed new light on treatment effectiveness and provide useful information regarding the conditions under which treatment may be most effective. Future directions for research in this area are discussed.
\end{abstract}

Keywords: Meta-analysis, Quantitative review, Childhood sexual abuse, Treatment outcomes

\section{Contents}

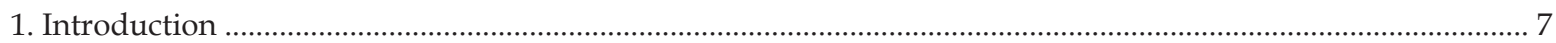

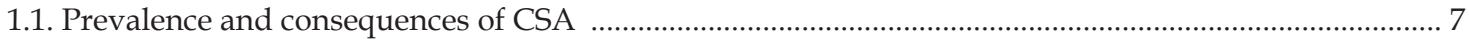

1.2. Evaluation of treatment for the sequelae of childhood sexual abuse .................................................. 7

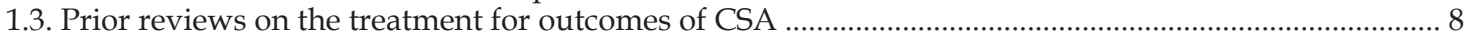

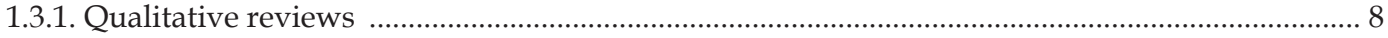

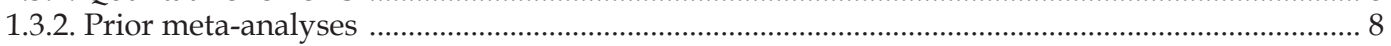

1.4. Importance of examining treatment and participant factors relevant to treatment effectiveness ..........8

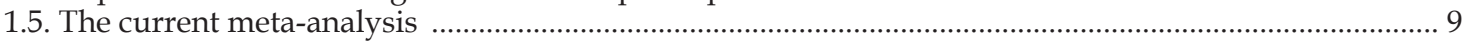

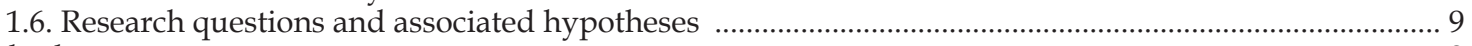

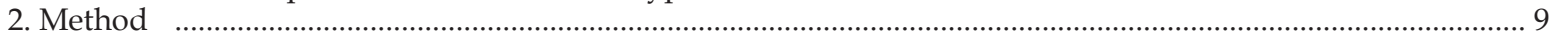

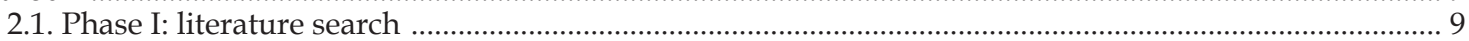

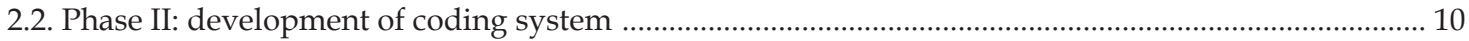

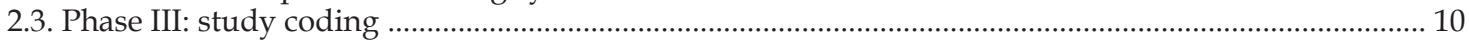

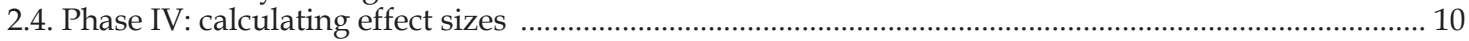

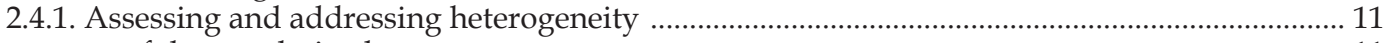

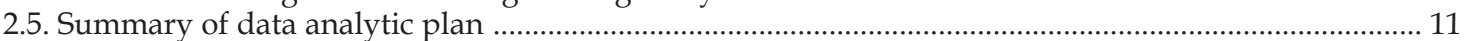

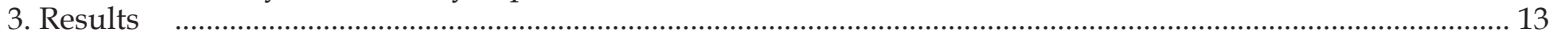

3.1. Treatment effects for outcomes of CSA using single-group pretest-posttest designs ........................ 13

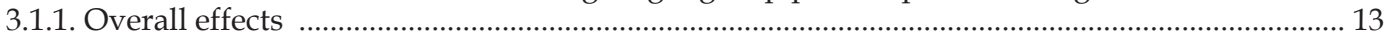

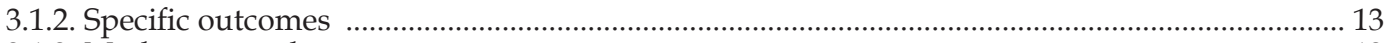

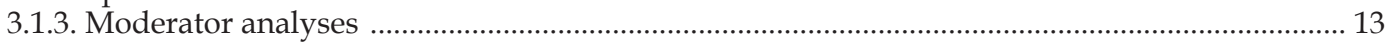

3.2. Treatment effects for outcomes of CSA using between-group designs ............................................ 13

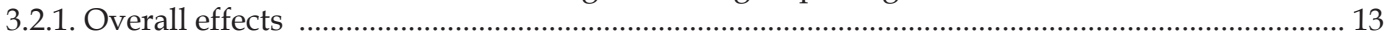

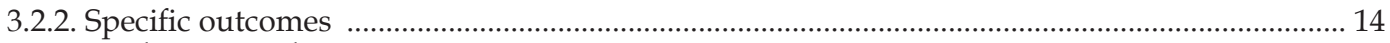

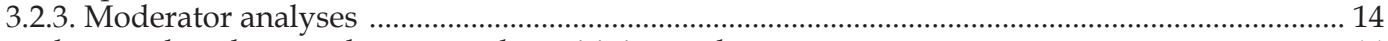

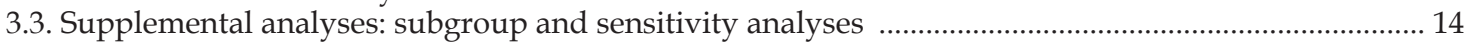

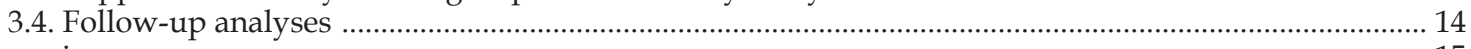

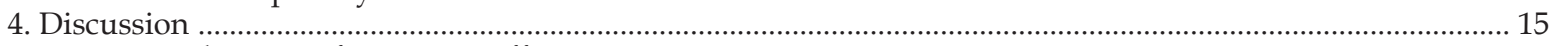

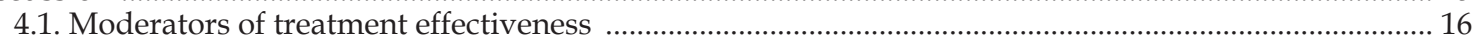

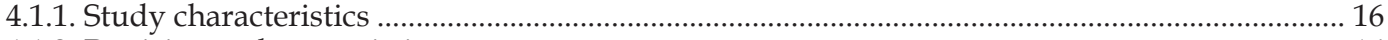

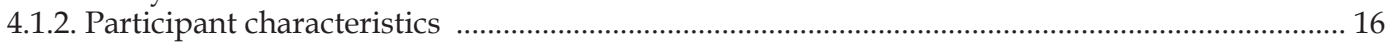

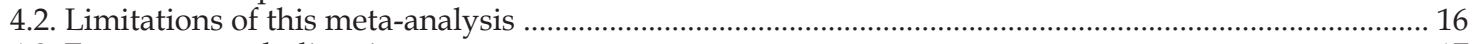

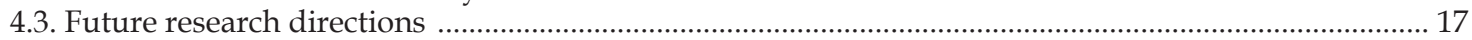

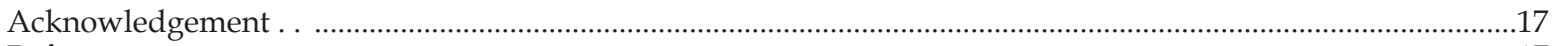

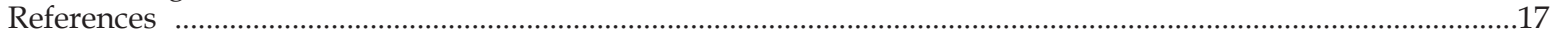




\section{Introduction}

Childhood sexual abuse is recognized as a distinct form of maltreatment with unique interpersonal characteristics (e.g., boundary violations, betrayal, sexual traumatization, stigma, and secrecy) that result in developmental consequences not associated with other forms of maltreatment (Noll, 2008). As such, a large body of literature has documented negative psychological outcomes for sexually abused children (KendallTackett et al., 1993., 2001). There is also evidence that CSA is more strongly linked to later mental health problems than other forms of abuse (Fergusson, Boden, \& Horwood, 2008). Finally, although CSA is experienced more often by females, males are also sexually abused and suffer similar negative consequences (Romano \& DeLuca, 2001).

In response to these problems, practitioners and applied researchers have developed and evaluated interventions to treat children who have experienced sexual abuse. Although individual treatment outcome studies and reviews have been published evaluating treatment gains associated with interventions for sequelae of CSA, there is need for an up-to-date comprehensive examination of the treatment outcome literature for problems experienced by victims of childhood sexual abuse (CSA). Thus, the purpose of the present study is to quantify the effectiveness of treatments for the most frequent outcomes of sexual abuse.

\subsection{Prevalence and consequences of CSA}

A recent meta-analysis of the international literature found that approximately $20 \%$ of women and $8 \%$ of men experience sexual abuse as children (Pereda, Guilera, Forns, \& GomezBenito, 2009). Although an encouraging trend indicates that sexual abuse in the U.S. appears to have declined in recent years (Jones, Finkelhor, \& Halter, 2006), the sexual victimization of children remains a significant problem, both in the U.S and internationally (Pereda et al., 2009). While not every sexually abused child experiences clinically significant symptomatology following abuse, (Kendall-Tackett et al., 1993; Sawyer, 2007), data are clear that a large proportion of sexually abused children and adolescents do experience deleterious outcomes.

The vast majority of documented outcomes fall into the categories of: trauma and PTSD-related symptomatology, externalizing behaviors, and internalizing problems (Kendall-Tackett et al., 2001; Putnam, 2003; Stevenson, 1999). Many of these outcomes may be more strongly associated with CSA, compared to other forms of maltreatment. For instance, there is evidence that sexually abused children are more likely to exhibit unique difficulties such as PTSD (e.g., Dubner \& Motta, 1999) and sexual behavioral problems (e.g., Adams et al., 1995; Trickett and McBride-Chang, 1995). Further, the research regarding the effects of CSA may be more consistent than for other types of abuse (Fergusson et al., 2008). This supports the necessity of examining treatment for the outcomes of CSA separately from other types of maltreatment.

PTSD is the most commonly diagnosed disorder among child victims of sexual abuse (Weinstein, Staffelbach, \& Biaggio, 2000). Estimates suggest that approximately 37\% to $53 \%$ of sexually abused children eventually develop PTSD (e.g., Kendall-Tackett et al., 1993; McLeer et al., 1988, 1998), and the large majority of sexually abused children referred to treatment have been shown to experience partial PTSD symptoms (McLeer, Deblinger, Henry, \& Orvaschel, 1992). Sexual abuse has been associated with traumatic reactions that may include re-experiencing the abuse through memories or dreams and actively attempting to avoid situations or stimuli that remind them of the abuse. Moreover, high prevalence of PTSD is unique to sexually abused children compared to children who have experienced other types of adversity (e.g., Tremblay, Hebert, \& Piche, 2000), which may explain why many studies investigating treatments for outcomes of CSA view reducing PTSD as a particularly desirable outcome.

In response to high levels of emotional distress stemming from the abuse, sexually abused children also may engage in a number of "acting out" behaviors (Nalavany, Ryan, \& Hinterlong, 2009). Indeed, externalizing problems, particularly sexual behavioral problems, hyperactivity, and aggression, are commonly reported among children with a history of CSA. More specifically, approximately $28 \%$ of sexually abused children exhibit highly sexualized behavior (Kendall-Tackett et al., 1993), which is one of the most widespread and troublesome problems reported following sexual abuse (Gray, Pithers, Busconi, \& Houchens, 1999). Another externalizing problem frequently diagnosed among sexually abused children includes Attention Deficit/Hyperactivity Disorder (ADHD; e.g., Weinstein et al., 2000). Aside from the diagnosis of ADHD per se, studies have shown that sexually abused children are significantly more hyperactive and aggressive than are non-maltreated children (e.g., Dubowitz et al., 1993; Swanston et al., 2003), and researchers have consistently found a high prevalence of conduct disorder in sexually abused children (e.g., Dubowitz et al., 1993; Lynskey and Fergusson, 1997; Romano et al., 2006).

CSA has also been linked to difficulties at the other end of the behavioral spectrum in the form of internalizing disorders such as depression and anxiety (Chaffin, Silovsky, \& Vaughn, 2005). For instance, sexually abused children display higher rates of depression than do non-abused children (e.g., Dubowitz et al., 1993), with as many as $43 \%$ to $67 \%$ of children meeting diagnostic criteria for depression following sexual abuse (e.g., Koverola et al., 1993; Tebbutt et al., 1997). Further, prevalence of anxiety disorders (e.g., phobias, separation anxiety disorder, and obsessive-compulsive disorder) is significantly higher in sexually abused children than in non-abused children (12\% versus 3\%; Spataro, Mullen, Burgess, Wells, \& Moss, 2004).

Evidence suggests that these disorders do not occur in isolation among sexual abuse victims, but instead are experienced as comorbid conditions. In fact, it is estimated that approximately $55 \%$ of children referred for treatment following sexual abuse meet criteria for more than one diagnosis (Target \& Fonagy, 1996). Further, negative outcomes of CSA often extend into adulthood and include substance abuse, suicidality, interpersonal problems, PTSD, depression, anxiety and anger (for a review see Chen et al., 2010; Neumann et al., 1996) Many of these outcomes occur regardless of the gender or age of the victim at the time of the abuse (Chen et al., 2010). Because the negative correlates of CSA are often long-term, early intervention with children is important to reduce the prevalence of adulthood problems.

\subsection{Evaluation of treatment for the sequelae of childhood sex- ual abuse}

Recognition of the widespread negative impact of CSA has driven efforts to develop and evaluate treatments that ameliorate these difficulties. While some of these studies are conducted in the context of highly-controlled environments (efficacy trials), others are conducted in clinical settings (effectiveness trials; Kazdin, 2003). Many studies, however, lie somewhere on a continuum between efficacy and effectiveness, depending on the amount of experimental control that is exerted in the study (Hoagwood, Hibbs, Brent, \& Jensen, 1995). Here, we use the term "effectiveness" generically to describe studies making up this body of literature. Represented within this literature are various research designs, including 
single-group pretest-posttest, quasi-experimental, and randomized controlled trials. Evaluation of the effectiveness of interventions for outcomes of CSA, regardless of design, is imperative given that the resources used to treat child abuse are limited and should be used to support the most successful interventions (Hansen, Warner-Rogers, \& Hecht, 1998). To date, several qualitative and meta-analytic reviews have sought to evaluate the effectiveness of these varying treatments.

\subsection{Prior reviews on the treatment for outcomes of CSA}

\subsubsection{Qualitative reviews}

A number of qualitative reviews examining treatment effectiveness for outcomes of CSA have been published. Among the most highly cited qualitative reviews are Finkelhor and Berliner (1995), Putnam (2003), Saywitz et al. (2000), and Stevenson (1999). A general consensus among these reviews is that treatment for outcomes of CSA is effective - and while not every child improves, overall, children show significant symptom reduction following treatment as compared to pretreatment scores or control groups. For instance, Stevenson (1999) concluded that within single-group pretest-posttest designs, participants demonstrate consistent improvement in the areas of self-esteem, anxiety, and depression following treatment. These reviews also concluded that too few randomized controlled trials have been conducted to definitely state that symptom reduction is due to treatment and not simply the passage of time. Moreover, treatment effectiveness appears to vary depending on research design. Single-group pretestposttest designs and randomized controlled designs produced consistent findings that almost all sexually abused children improve significantly after the completion of treatment; however, quasi-experimental designs produced less consistent results. Lastly, two of the reviews (Putnam; Saywitz et al.) concluded that abuse-specific cognitive-behavioral therapy (CBT) was more effective than other treatments.

\subsubsection{Prior meta-analyses}

Although some meta-analyses (e.g., Silverman et al., 2008) have examined treatment efficacy for trauma exposure broadly defined (i.e., including CSA, exposure to parental violence, and motor vehicle accidents), five published metaanalyses have reviewed the literature on treatment for outcomes associated specifically with CSA (i.e., Hetzel-Riggin et al., 2007; Macdonald et al., 2006; Reeker et al., 1997; Skowron and Reinemann, 2005). Although each of these studies has made unique contributions, limitations of these investigations reduce the generalizability of findings to sexually abused children outside of their respective samples. In the earliest of these reviews, Reeker et al. examined the effectiveness of group treatments for CSA victims using the following outcomes: internalizing symptoms, externalizing symptoms, sexual behaviors, and self-esteem. This meta-analysis focused on 15 initial studies published between 1986 and 1996 with a total $n$ of 220. Results revealed a large mean effect size of $d=.79$. Although this study was among the first to synthesize findings regarding treatment outcomes for sequelae of CSA, it examined only group treatments that utilized a single-group design, which may produce an inflated effect size due to the lack of control groups to account for the passage of time (Lipsey \& Wilson, 2001). Further, at least 19 treatment outcome studies for outcomes of sexual abuse have been published since this study was published in 1996. These investigations are included in this updated meta-analysis.

More recently, Skowron and Reinemann (2005) conducted a meta-analysis examining the effects of psychological interventions for outcomes related to three forms of child maltreatment, including sexual abuse, physical abuse and ne- glect. These authors examined the impact of interventions on the following outcomes: internalizing problems, externalizing problems, and cognitive processes. Although 21 studies were included in this meta-analysis, only seven of these studies, with a total participant sample size of $n=397$, specifically addressed outcomes associated with CSA. Further, only randomized controlled designs published before 2000 were examined. Results revealed a mean difference effect size of $d=.69$, which suggests that treatment conditions resulted in greater improvements relative to comparison conditions (Skowron \& Reinemann). Lastly, although this meta-analysis took the important step of examining the impact of treatment characteristics (e.g., modality, quality of study design) on general maltreatment interventions, additional information may be gleaned by also exploring the role of participant characteristics such as gender, age, or ethnicity in relation to treatment for CSA outcomes specifically.

Macdonald et al. (2006), a Cochrane systematic review, included 10 studies with a total participant sample size of $n=847$. This meta-analysis examined the impact of cognitivebehavioral treatment compared to control groups on child psychological functioning, child behavior problems, and parenting skills and knowledge (e.g., belief in their child's story). Results revealed that although CBT interventions significantly reduced children's PTSD symptoms and anxiety, there were no significant reductions in child depression or behavior problems compared to the control groups. This meta-analysis provides important data addressing the effectiveness of CBT interventions. However, relatively few studies $(N=9)$ were available for inclusion given that single-group pretest-posttest and quasi-experimental designs were excluded from the analysis. Further, the authors compared cognitive-behavioral treatments to a combined control group consisting of actual control conditions and treatment as usual conditions. Combining these two types of control groups may cloud conclusions about the relative efficacy of CBT compared to either a true control group or another type of treatment. Finally, although evaluating cognitive-behavioral treatments is certainly of interest given their relative prevalence in the literature, it is also important to compare the differential effectiveness of cognitive-behavioral and other treatments utilized for outcomes of CSA (e.g., EMDR).

Hetzel-Riggin et al. (2007) was the most recent meta-analysis, which included 28 studies with a participant sample size of $n=1839$, found that psychological treatment for CSA outcomes was significantly more effective than no treatment (Hetzel-Riggin et al., 2007). While both single- and between-group designs were included in this analysis, findings were analyzed using pretest-posttest meta-analytic methods, which did not include comparisons to control or other treatment conditions. Utilizing a between-group statistical approach would also allow for an examination of between-group differences (e.g., CBT versus supportive therapy). Finally, some studies included in the meta-analysis did not explicitly focus on evaluating the effectiveness of treatment for sequelae of CSA, which makes generalizability difficult to determine. For instance, one study combined participants with a history of CSA, a history of same-age peer rape, and no history of sexual victimization (Krakow et al., 2001).

\subsection{Importance of examining treatment and participant fac- tors relevant to treatment effectiveness}

Although examining the overall effectiveness of treatments for sequelae of CSA is critical for advancing treatment, it is equally important to understand the conditions under which treatment is more or less effective. Examination of treatment moderators is important because it may lead to a deeper un- 
derstanding of how specific interventions work (Kazdin, 2003). More specifically, such knowledge can help identify subpopulations for which there are different mechanisms of change, providing unique and important information beyond traditional treatment effectiveness analyses (Kraemer, Wilson, Fairburn, \& Agras, 2002). Such understanding of treatment mechanisms, in turn, may be the best investment for improving clinical practice (Kazdin \& Nock, 2003). A recent article examining psychosocial treatments for trauma suggests that future research should examine variables, such as dose effect and age, to determine whether they may moderate treatment effects (Silverman et al., 2008).

Thus, a second goal of the present study was to investigate treatment and participant characteristics that may impact the effectiveness of treatment. Prior meta-analyses reveal that nearly $22 \%$ of the variance in treatment outcomes may be due to treatment characteristics, including theoretical orientation, type of study design, length of treatment, and treatment modality (Lipsey, 1992). For instance, theoretical approach may have a bearing on treatment outcomes (e.g., Jaberghaderi, Greenwald, Rubin, Zand, \& Dolatabadi, 2004). Indeed, cognitive-behavioral therapy has been shown to be more effective than supportive therapy (an attention-placebo) in reducing sexual acting out among sexually abused children (Cohen \& Mannarino, 1997). Further, when a control group is used to investigate treatment outcomes for CSA victims, effect sizes are smaller than with a single-group pretest-posttest design. Moreover, lengthier treatment may be needed to decrease negative outcomes for some children (Hetzel-Riggin et al., 2007). In addition, recent evidence (Skowron \& Reinemann, 2005) suggests that no differences in effect sizes may exist between various treatment modalities (e.g., group versus individual). However, confirming these findings in a comprehensive metaanalysis (e.g., including all available studies across multiple research designs) is necessary to more definitively understand this association.

A number of participant factors, including age, ethnicity, and gender, also may be important in understanding the conditions under which treatment is most effective. Age is an important factor to consider when assessing treatment outcomes because cognitive abilities that develop with age may make older children more responsive to cognitively-based interventions. Past research has found greater treatment gains for studies including more non-Caucasian participants (Hetzel-Riggin et al., 2007). Gender also may impact treatment outcomes. For example, although the Reeker et al. (1997) meta-analysis on group treatments for outcomes of CSA $(n=15)$ did not find significant differences in effect sizes based on gender, there was a trend (i.e., females $d=.96$, males $d=.30$ ) for studies with female participants to demonstrate larger effect sizes.

\subsection{The current meta-analysis}

This current study was designed to assess overall effectiveness of treatments for the negative consequences of CSA as well as the specific conditions under which treatments might be more or less effective. This meta-analysis includes all studies written in English from 1980 to 2009 that included participants younger than 18 who were being treated for any of the three most prominent sequelae of CSA: PTSD, internalizing problems, or externalizing behaviors (including sexualized behavior). This meta-analysis is an improvement on prior examinations of this kind because it allowed for the investigation of the impact of multiple treatment modalities (e.g., group, individual treatments), research designs (e.g., single-group pretest-posttest designs, between-group designs), and theoretical approaches to treatment (e.g., CBT, play therapy). Further, although two meta-analyses included a limited number of un- published dissertations (Macdonald et al., 2006; Skowron and Reinemann, 2005), the present study includes all unpublished dissertations. Because the effects reported in published studies are often higher than those reported in unpublished studies (Begg, 1994), the comprehensive inclusion of unpublished dissertations should reduce publication bias in the present analysis.

\subsection{Research questions and associated hypotheses}

Understanding whether treatment for sexually abused children is effective is crucial given the high prevalence rates of abuse and the negative outcomes that victims often experience. However, as noted earlier, the summative limitations of past reviews are such that a thorough and up-to-date metaanalysis is needed to evaluate the treatments for CSA outcomes as well as the conditions under which treatments may be more or less effective. Toward that end, we propose the following research questions and hypotheses:

1. Is treatment for the well-established outcomes of CSA effective? Treatments as a whole were expected to demonstrate effectiveness [operationalized as a medium to large effect size $(d>.5)$ ] across the most common negative outcomes associated with CSA: PTSD symptoms, externalizing problems, and internalizing problems.

2. Do specific treatment characteristics moderate the effects of interventions for children experiencing negative outcomes associated with CSA? Based on research showing that a cognitive-behavioral approach to treatment may be more effective than other theoretical approaches (Cohen and Mannarino, 1997; Macdonald et al., 2006) and that receiving more treatment sessions is more effective than receiving fewer sessions (Hetzel-Riggin et al., 2007), we expected that both cognitive-behavioral and longer treatments would be more effective. We also expected that singlegroup pretest-posttest study designs would yield greater effect sizes than would between-group designs. Finally, group and individual treatments were not expected to differ with regard to effect sizes.

3. Do key participant characteristics impact treatment outcomes? Based on past research (Deblinger, Stauffer, \& Steer, 2001), treatment was expected to be more effective for older children. Further, past meta-analyses (HetzelRiggin et al., 2007; Reeker et al., 1997) suggest that treatment may be more effective for non-Caucasian and female participants.

\section{Method}

Meta-analysis involves gathering and coding research studies, then analyzing the resulting data to describe patterns of findings in the sample of studies. This meta-analysis was conducted in four phases, which are outlined later.

\subsection{Phase I: literature search}

The first step involved specifying clear inclusion criteria to search the relevant literature. For the present project, studies published between 1960 and December 2009 that satisfied the following criteria were included: each study explicitly focused on evaluating the effects of a treatment for the sequelae of CSA experienced by victims under 18 years of age; each study must have been written in English; each study must have assessed the effectiveness of an intervention using at least one outcome that could be categorized as either PTSD symptoms, externalizing problems, or internalizing problems; each study 
must have provided statistical information for the calculation of effect sizes or if this information was missing the authors were contacted and must have provided this information; and each between-group study must have included a no-treatment or attention-placebo comparison group. Studies published in other languages, that included participants over the age of 18 , that only included case studies, that did not specifically focus on evaluating treatment for the outcomes of CSA, or that did not include a clear no-treatment or attention-placebo comparison group were excluded from this meta-analysis. A thorough examination of social science citation retrieval systems (i.e., Cochrane Database of Systematic Reviews, Dissertation Abstracts International, Eric, MEDLINE, ProQuest Dissertations and Theses, PsycINFO, the Social Science Citation Index, and Sociological Abstracts) was conducted. Several combinations of keywords (e.g., child abuse, sexual abuse, child maltreatment, treatment, intervention, and therapy) were used to identify relevant studies. Further, a thorough search of the references in each relevant article and qualitative review as well as a manual search of journals relevant to CSA (e.g., Journal of Child Sexual Abuse, Child Abuse and Neglect, Child Maltreatment, and Journal of Interpersonal Violence) was conducted to ensure that all relevant studies were included in this meta-analysis. This search identified 35 articles or dissertations that met the inclusion criteria outlined earlier.

\subsection{Phase II: development of coding system}

Another crucial step in conducting a meta-analysis is the development of a comprehensive coding system, which reflects all variables of interest in the study. This stage consisted of developing an all-inclusive list of variables that corresponded with the primary research questions of the project. In general, there were three classes of variables: psychological outcomes, which consisted of PTSD, externalizing (i.e., ADHD, Oppositional Defiant Disorder/Conduct Disorder, sexual behavior problems, and aggression), and internalizing problems (i.e., anxiety, depression); Treatment characteristics, which included theoretical approach to treatment (cognitive-behavioral versus other theoretical orientations), treatment duration (average length of treatment in weeks), inclusion of caregiver in treatment (yes/no), publication type (dissertation versus published article), type of control group (no-treatment comparison, wait-list comparison, minimal contact, attention-placebo, or treatment as usual), study design (single-group pretest-posttest, quasi-experimental, and randomized controlled trial), treatment modality (group, individual, family, or combination approaches); and participant characteristics including age, gender, and ethnicity. For the psychological outcomes, the means, standard deviations, and sample sizes were recorded for each treatment condition when available. T-tests, F-tests, correlations, and p-values also were recorded if provided. The coding system served to enhance the reliability of the study by increasing intercoder agreement among coders.

\subsection{Phase III: study coding}

Coding of all studies was completed by the first author. Further, the second author coded a randomly selected $25 \%$ of the articles using a coding manual developed for this study. The first and second authors achieved high overall intercoder agreement across all variables on the random sample of studies $($ kappa $=.80)$. Interrater reliabilities were calculated individually for select moderator variables that were either subject to misinterpretation or posed coding difficulties. Kappas were above .90 for research design, theoretical orientation, ethnicity, and treatment modality, and the intraclass correlation coefficient for the length of treatment was 1.0. Interrater reliabil- ities were not calculated for moderator variables such as age or gender, due to lack of subjectivity in coding these variables.

\subsection{Phase IV: calculating effect sizes}

Effect sizes were calculated using the meta-analytic software, Comprehensive Meta-Analysis (CMA; Borenstein, Hedges, Higgins, \& Rothstein, 2005). In addition to computing effect sizes, CMA also performs sensitivity analyses, which examine the impact of outliers on the results, and tests for moderating relationships, which explain variability in effect sizes between studies. Lastly, CMA creates funnel plots to investigate the presence of publication bias (e.g., the tendency for studies with significant results to be published over studies with non-significant results, which makes them easier to locate).

Separate meta-analyses were conducted for: a) singlegroup pretest-posttest designs, and b) quasi-experimental designs and randomized controlled trials, because effect sizes from within- and between-group designs may be incompatible (Lipsey \& Wilson, 2001). Single-group designs (i.e., withinsubjects) lack a control group to account for the passage of time, often leading to effect sizes that are greater in magnitude and should therefore not be mixed in the same meta-analysis as effect sizes from randomized controlled trials (Lipsey \& Wilson, 2001). To confirm that conducting separate metaanalyses was appropriate, a subgroup analysis was conducted comparing effect sizes from single-group pretest-posttest designs and between-group designs (Morris \& DeShon, 2002). This subgroup analysis indicated that the average singlegroup pretest-posttest effect size was larger than the average between-group design effect size.

For single-group pretest-posttest designs, a standardized mean gain effect size was calculated, which measures change over time. For quasi-experimental and randomized controlled designs a standardized mean difference effect size was calculated, which examines differences between groups on mean values. These standardized effect sizes were classified according to the guidelines set forth by Cohen (1988), as small $(d=.2-.5)$, medium $(d=.5-.8)$, or large ( $d=.8$ or above). An effect size of $d=1$ translates to a change of one standard deviation either over time (i.e., standardized mean gain effect size), or between treatment and comparison groups at posttest (i.e., standardized mean difference effect size).

For studies in which means and standard deviations were not reported ( $n=4$ pre-post studies; i.e., Brown, 2007; De Luca and Hazen, 1993; Hall-Marley and Damon, 1993; Monck, 1997), the effect size was estimated. For instance, if means were missing from a study utilizing a single-group pretestposttest design, a standardized mean gain effect size was estimated using a paired sample $t$-test, a pre-post correlation, and the sample size:

$$
d= \pm t \sqrt{\frac{2(1-r)}{n}}
$$

When sufficient information was not included in the study to calculate effect sizes, the authors were contacted in an attempt to obtain this statistical information. The response rate was 33\% (one out of three authors responded) and resulted in excluding two studies due to a lack of response from the authors. Further, four studies contributed only partial data to the meta-analysis due to no response from the authors or missing data (e.g., data from the BASC was included but not the Children's Depression Inventory). However, funnel plots did not indicate that publication bias a problem within this analysis.

An effect size was computed for each applicable outcome (i.e., PTSD symptoms, externalizing behaviors, and internaliz- 
ing problems) within studies. Many studies contributed more than one effect size representing the same construct, (e.g., depression) to each meta-analysis. These effect sizes were averaged creating one effect size per construct, which facilitated statistical independence of the data (Lipsey \& Wilson, 2001).

\subsubsection{Assessing and addressing heterogeneity}

Initially, the effect sizes were analyzed using both fixed and random effects modeling, which are procedures used by well-accepted meta-analytic approaches such as the Cochrane Collaboration (Higgins \& Green, 2009). A formal test of heterogeneity using the $\mathrm{I}^{2}$ index was preformed, which assessed the percent of variance in effect size estimates that is due to between study heterogeneity and not sampling error within studies. In this study a high rate of heterogeneity across effect sizes existed, and therefore to calculate effect sizes, the DerSimonian and Laird approach was used for the random effects modeling of both continuous and dichotomous data (Higgins \& Green, 2009). A random effects approach assumes that the true effect size varies from study to study (Sheu \& Suzuki, 2001). With a random effects framework, the average mean weighted effect size is the estimate of the mean of the distribution of effect sizes. Studies in this meta-analysis differed by age, type of treatment utilized, and other key variables. Thus, effect sizes from each study were expected to differ, which is consistent with a random effects modeling approach to calcu- lating effect sizes (Gliner, Morgan, \& Harmon, 2003). In fact, tests of heterogeneity were significant and remained significant when using random effects modeling. This suggests that, in addition to sampling error, systematic factors can explain the variance in the effect sizes. Given the high rate of heterogeneity within each meta-analysis, moderating analyses were conducted to identify possible sources of systematic differences across studies.

To examine potential moderators (consistent with research questions 2 and 3 ) that may account for variability across studies (i.e., heterogeneity), two types of analyses were conducted. Subgroup analyses, which are analogous to Analysis of Variance (ANOVA), were conducted for categorical variables (e.g., Is treatment more effective for females than for males?). Further, meta-regression analyses were conducted for continuous moderator variables (e.g., Does the treatment effect increase as a function of the length of treatment?). The subgroup analyses consisted of calculating a $Q_{\mathrm{b}}$ statistic, which assessed for heterogeneity between effect sizes (Lipsey \& Wilson, 2001).

\subsection{Summary of data analytic plan}

Consistent with the primary research questions identified in this study, the data analytic plan entailed four stages (see Figure 1) conducted separately for a) single-group pretestpost test designs, and b) between-group designs.

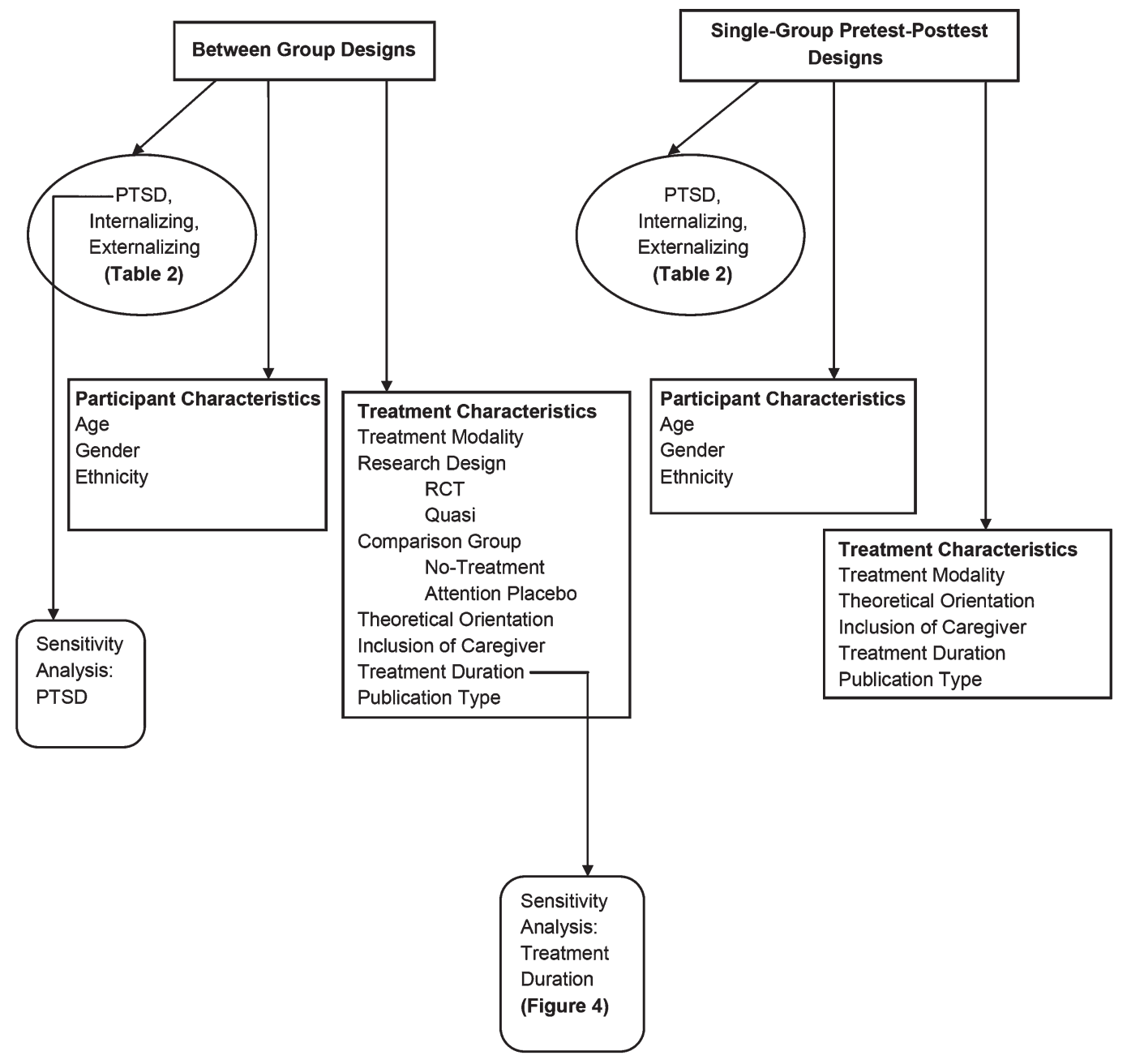

Figure 1. Meta-analytic design. 
Table 1. Study characteristics.

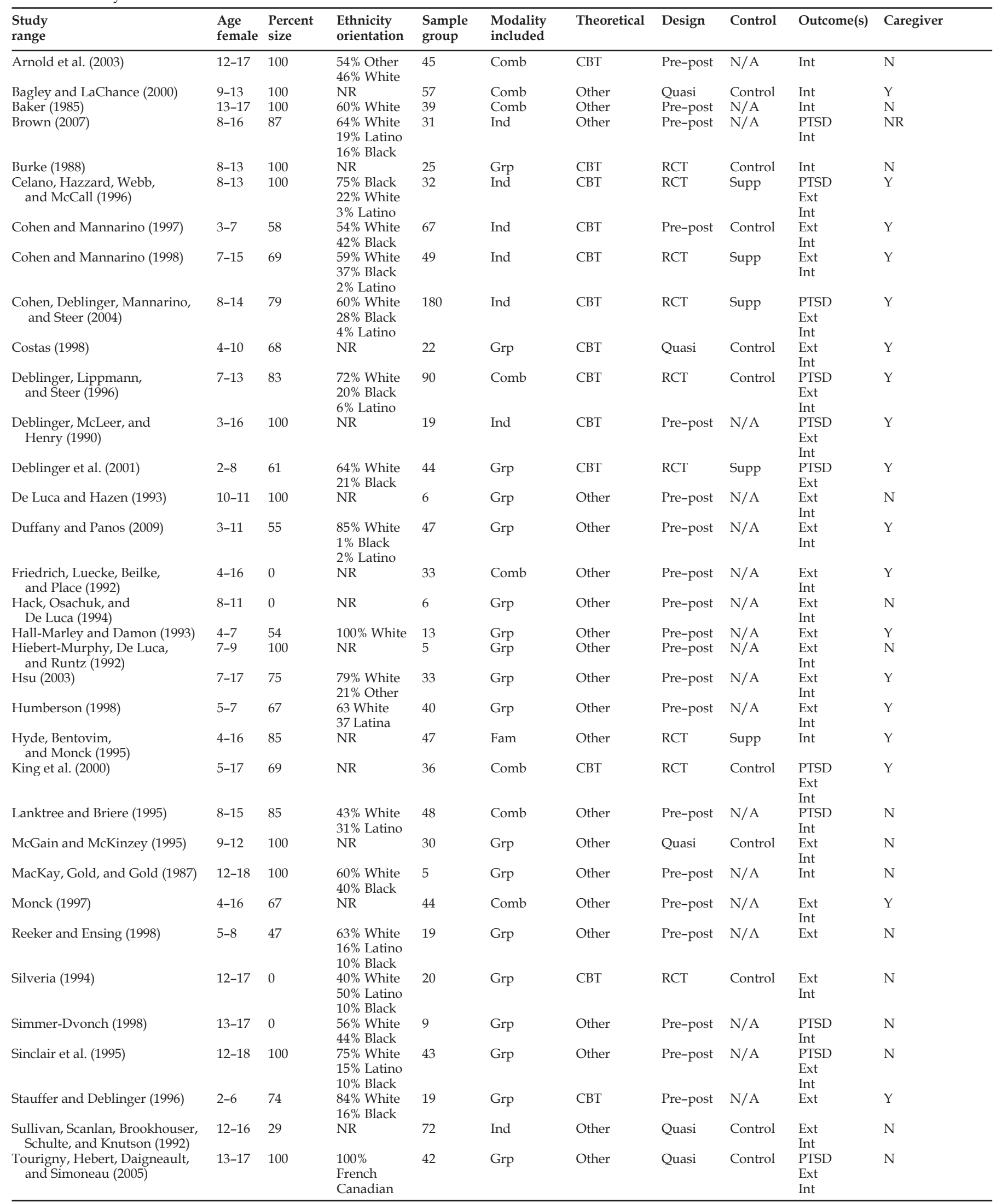

For modality, Ind = individual therapy; Grp = group therapy; Fam = family therapy; Comb = combination of two or more treatment modalities. For type of control group, Control = no-treatment, wait-list control, or minimal contact; Supp = supportive therapy or attention-placebo. $\mathrm{Y}=\mathrm{yes}$; $\mathrm{N}=$ no. $\mathrm{NR}$ indicates that this information was not reported. $\mathrm{N} / \mathrm{A}=$ not applicable. 
Funnel Plot of Standard Error by Std diff in means

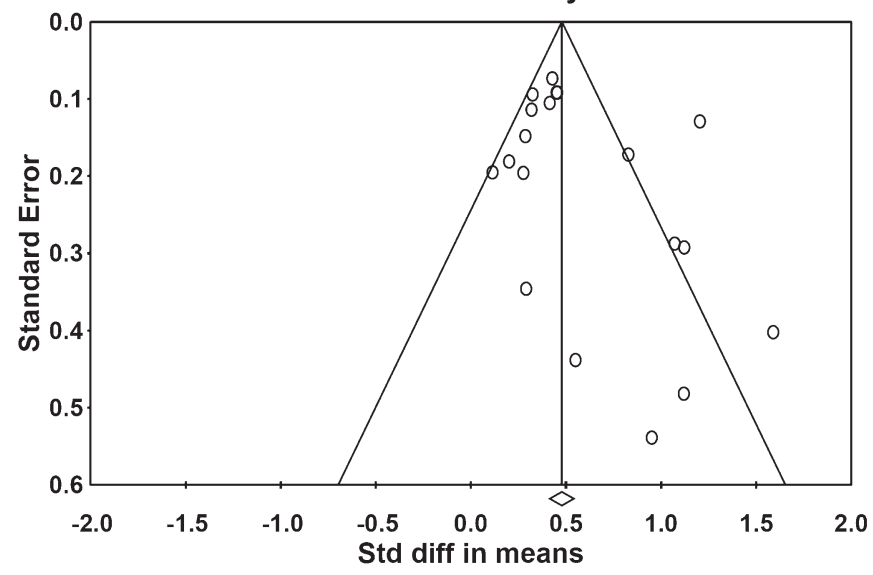

Figure 2. Funnel plot of effect sizes by standard error for studies utilizing a single-group pretest-posttest design.
Table 2. Weighted mean effect sizes by outcome and research design.

\begin{tabular}{|c|c|c|c|c|c|c|}
\hline Outcome & $\mathrm{K}^{\mathrm{a}}$ & $\begin{array}{l}\text { Effect } \\
\text { size }\end{array}$ & $S E$ & $95 \% \mathrm{CI}$ & $\begin{array}{c}\text { Fail-safe } \\
\text { Ns }\end{array}$ & $\mathrm{I}^{2}$ \\
\hline \multicolumn{7}{|l|}{ Overall } \\
\hline Pre-post ${ }^{b}$ & 19 & $.54^{* *}$ & .07 & $.40-.68$ & 1005 & $73.88^{* *}$ \\
\hline Between-group designs & 16 & $.54^{* *}$ & .11 & $.33-.76$ & 438 & $79.00^{* *}$ \\
\hline \multicolumn{7}{|c|}{ PTSD } \\
\hline Pre-post ${ }^{b}$ & 5 & .51 & .27 & $-.01-1.03$ & 348 & $91.14^{* *}$ \\
\hline Between-group designs & 6 & $.63^{* *}$ & .19 & $.26-1.00$ & 57 & $64.25^{*}$ \\
\hline \multicolumn{7}{|l|}{ Externalizing } \\
\hline Pre-post ${ }^{b}$ & 14 & $.47^{* *}$ & .08 & $.31-.64$ & 304 & $61.30^{* *}$ \\
\hline Between-group designs & 12 & $.39^{*}$ & .17 & $.06-.70$ & 60 & $76.40^{* *}$ \\
\hline \multicolumn{7}{|l|}{ Internalizing } \\
\hline Pre-post ${ }^{b}$ & 16 & $.50^{* *}$ & .06 & $.39-.61$ & 671 & $50.99^{*}$ \\
\hline Between-group designs & 15 & $.56^{* *}$ & .12 & $.33-.80$ & 274 & $72.31^{* *}$ \\
\hline
\end{tabular}

a. Number of studies.

b. Single-group pretest-posttest designs.

${ }^{* *} p<.01 ; * p<.05$
1) Conducted overall cumulative meta-analyses and tests of heterogeneity for each outcome of CSA: PTSD symptoms, externalizing problems, and internalizing problems.

2) Conducted moderator analyses for participant and treatment characteristics discussed earlier following significant tests of heterogeneity for overall cumulative meta-analyses.

3) Examined supplemental subgroup and sensitivity analyses to elucidate inconsistent findings among the single-group pretest-posttest and between-group moderator results.

\section{Results}

\subsection{Treatment effects for outcomes of CSA using single-group pretest-posttest designs}

\subsubsection{Overall effects}

This meta-analysis of pretest-posttest study designs examined the overall effects of treatment following CSA, and included a total of 493 participants from 19 studies (Ns range $=5$ to 48). Table 1 provides additional descriptive information detailing the major characteristics of each study. The test of heterogeneity indicated that there was significant variability $\left[\mathrm{I}^{2}\right.$ $(18)=73.88, p<.01]$ across the effect sizes included in this meta-analyses. Therefore, random effects modeling was used to calculate the effect sizes and moderating analyses were conducted to investigate potential systematic differences between studies. According to Cohen (1988), the overall mean weighted effect size for the meta-analysis was of medium strength, $d=.54, p<.01(95 \%$ confidence interval $=.40-.69)$ and the effect sizes included in the analysis ranged from .12 to 1.59 . This meta-analysis suggests that treatment significantly reduced the negative outcomes of CSA compared to participant's pretreatment scores. To account for potential file-drawer problems, a fail-safe $N$ was calculated. The fail-safe $N$ was 1005 , indicating that 1005 studies would need to report null findings to decrease the overall mean effect size to non-significance. To confirm the findings of the fail-safe $N$, a funnel plot was generated (see Figure 2). A visual inspection uncovered a symmetrical funnel plot with numerous trials approaching a mean difference of zero, providing additional support for the robustness of the overall mean effect size.

\subsubsection{Specific outcomes}

Within single-group pretest-posttest designs the mean weighted effect sizes for PTSD symptoms $(d=.51, p=.055$, $n=5)$, externalizing $(d=.47, p<.01, n=14)$ and internalizing $(d=.50, p<.01, n=16)$ problems were medium, although the PTSD effect size was not significant. These meta-analytic results are summarized in Table 2 and suggest that in singlegroup pretest-posttest designs psychological treatment is effective in reducing levels of PTSD symptoms, externalizing, and internalizing problems following childhood sexual abuse.

\subsubsection{Moderator analyses}

These analyses investigated the relationship between effect sizes and study characteristics as well as participant characteristics within single-group pretest-posttest studies. All analyses were calculated using the total effect size. The results were not broken out by outcome because of the small study sample size. For study characteristics, no significant moderating effects were found for inclusion of caregiver in treatment $\left(Q_{\mathrm{b}}=.09, p=.77, n=19\right)$, treatment modality $\left(Q_{\mathrm{b}}=.22\right.$, $p=.64, n=19)$, treatment duration $\left(Q_{\mathrm{b}}=3.4, p=.06, n=19\right)$, or research design $\left(Q_{\mathrm{b}}=.43, p=.51, n=19\right)$. Due to small study sample size, cognitive-behavioral treatments were contrasted with "other" theoretical orientations. Treatment effects were significantly larger for cognitive-behavioral treatments, $Q_{\mathrm{b}}=21.59, p<.01 ; d=1.05, p<.01(95 \% \mathrm{CI}=.8-1.3$, $n=3)$ when compared to treatments utilizing other theoretical orientations, $d=.41, p<.01(95 \% \mathrm{CI}=.31-.51, n=16)$. Finally, published studies produced larger treatment effects $\left(Q_{\mathrm{b}}=8.50, p<.01 ; d=.67, p<.01 ; 95 \% \mathrm{CI}=.48-.86, n=14\right)$ when compared to unpublished dissertations $(d=.34, p<.01$; $95 \% \mathrm{CI}=.22-.46, n=5$ ). For participant characteristics, no significant moderating effects were found for ethnicity, $Q_{\mathrm{b}}=2.07$, $p=.36 ; n=14$, mean age, $Q_{\mathrm{b}}=.21, p=.65 ; n=11$, or gender, $Q_{\mathrm{b}}=.10, p=.75 ; n=19$.

\subsection{Treatment effects for outcomes of CSA using between- group designs}

\subsubsection{Overall effects}

This meta-analysis examined overall effectiveness of treatment following CSA and included a total of 852 participants from 16 studies (Ns range $=20$ to 180). Table 1 provides 


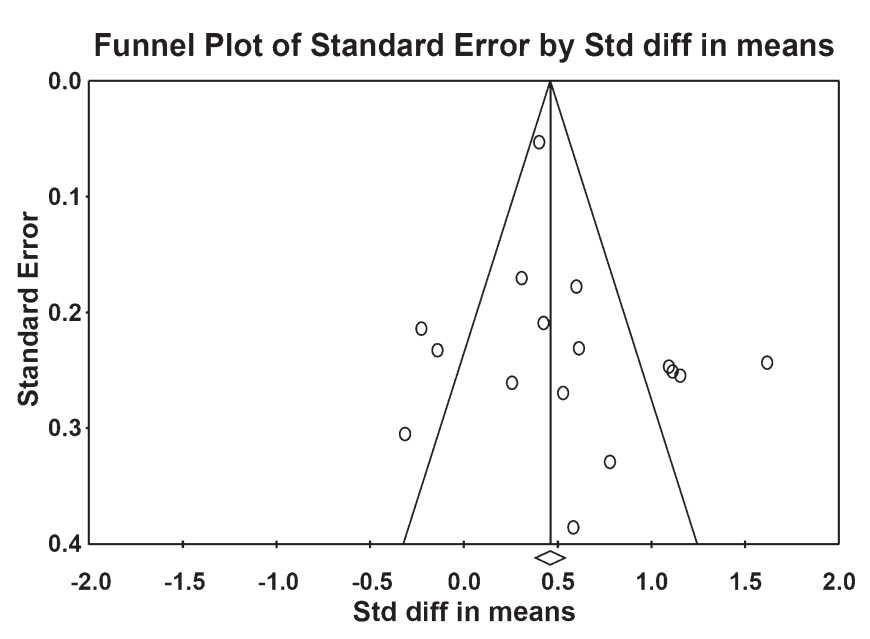

Figure 3. Funnel plot of effect sizes by standard error for studies utilizing a between-group design.

additional descriptive information detailing the major characteristics of each study. The test of heterogeneity indicated that there was significant variability $\left[\mathrm{I}^{2}(16)=79.00, p<.01\right]$ across the effect sizes included in this meta-analyses. Therefore, random effects modeling was used to calculate the effect sizes and moderating analyses were conducted to investigate potential systematic differences between studies. According to the guidelines set forth by Cohen (1988), the overall mean weighted effect size for the meta-analysis was of medium strength, $d=0.54, p<.01$ (95\% confidence interval $=.33-$ .76) and the effect sizes included in the analysis ranged from -.31 to 1.62. This meta-analysis provides evidence of the overall effectiveness of abuse-specific treatment in reducing the negative outcomes of CSA compared to no-treatment and attention-placebo (e.g., supportive therapy) comparison and control groups. To account for potential file-drawer problems, a fail-safe $N$ was calculated. The fail-safe $N$ was 438 , indicating that 438 studies would need to report null findings to decrease the overall mean effect size to non-significance. To confirm the findings of the fail-safe $N$, a funnel plot was generated (see Figure 3). Similar to the findings for single-group pretest-posttest designs, a visual inspection of the funnel plot uncovered a symmetrical funnel plot with trials around a mean difference of zero. This provides further support for the robustness of the overall mean effect size.

\subsubsection{Specific outcomes}

For between-group designs, the mean weighted effect size for the PTSD meta-analysis was medium $(d=.63, p<.01$, $n=6$ ). For externalizing problems, the mean weighted effect size for the meta-analysis was small $(d=.39, p<.05, n=12)$. Lastly, for internalizing problems, the mean weighted effect size for the meta-analysis was medium $(d=.56, p<.01, n=15)$. These meta-analyses provide consistent evidence that psychological treatment is effective in reducing PTSD symptoms, externalizing, and internalizing problems following childhood sexual abuse.

\subsubsection{Moderator analyses}

These analyses investigated the relationship between effect sizes and study characteristics as well as participant characteristics for between-group studies. For study characteristics, there were no significant moderating effects of treatment modality, $Q_{\mathrm{b}}=.54, p=.46, n=16$, type of research design, $Q_{\mathrm{b}}=.43, p=.51, n=16$, theoretical orientation, $Q_{\mathrm{b}}=.50$, $p=.48, n=16$, or publication type, $Q_{\mathrm{b}}=.39, p=.53, n=16$. However, there were significant moderating effects for type of comparison group, inclusion of caregiver in treatment, and treatment duration. Specifically, treatment effects were significantly larger for studies that contained a no-treatment comparison group, $Q_{\mathrm{b}}=6.70, p<.05 ; d=.79, p<.01$ (95\% confidence interval $=.45-1.13, n=9$ ), when compared to studies that contained an attention-placebo comparison group, $d=.25$, $p<.01$ (95\% confidence interval $=.03-.48, n=7)$. Further, effects were larger, $Q_{\mathrm{b}}=5.80, p<.05$, for studies that included only child participants, $d=.89, p<.01$ (95\% confidence interval $=.45-1.30, n=7$ ) than for studies that included both the caregiver and the child, $d=.31, p<.01$ (95\% confidence inter$\mathrm{val}=.14-.50, n=9$ ). Additionally, there was a positive effect of treatment duration such that longer duration was associated with larger treatment effects, $Q_{\mathrm{b}}=3.80, p=.05$.

For participant characteristics, no significant moderating effects were found for ethnicity, $Q_{\mathrm{b}}=.40, p=.53, n=11$. There was, however, a significant relationship between mean age and treatment effects, $Q_{\mathrm{b}}=4.28, p<.05, n=11$. Specifically, treatment effects increased as mean age increased. Further, treatment effects were significantly larger, $Q_{\mathrm{b}}=7.90, p<.01$, for the two studies that had a majority of male participants, $d=1.02, p<.01(95 \%$ confidence interval $=.62-1.40, n=2)$ compared to studies that had a majority of female participants, $d=.44, p<.01(95 \%$ confidence interval $=.36-.52, n=14)$.

\subsection{Supplemental analyses: subgroup and sensitivity analyses}

An examination of a scatter plot (see Figure 4a) uncovered an outlier in the treatment duration meta-analysis (i.e., treatment duration was 104 weeks for the Bagley and LaChance (2000) study compared to an average of 16.45 weeks for the other 15 studies). Therefore, a sensitivity analysis was conducted both with and without the Bagley study to assess its impact on the meta-analysis (see Figure $4 \mathrm{~b}$ ). This sensitivity analysis suggested that the Bagley study did not have a significant impact on the study as there was a positive effect for treatment duration even after excluding the Bagley study, $Q_{\mathrm{b}}=24.50, p<.01$. Therefore, this study was retained in the analyses.

\subsection{Follow-up analyses}

While a small number of studies investigated the longterm effectiveness of interventions, they did so using dissimilar follow-up periods resulting in too few studies to combine these data. However, a qualitative assessment of studies that did report follow-up data suggests that treatment gains were maintained up to a year after treatment was completed. For instance, Burke (1988) found that children continued to have significantly less anxiety and depression six weeks after treatment when compared to a wait-list control group. Similarly, three studies reported continued symptom reduction at a three month follow-up, both within subjects and compared to control groups (Deblinger et al., 2001; King et al., 2000; Stauffer and Deblinger, 1996). DeLuca and Hazen (1993) reported that while anxiety symptoms were significantly lower nine months following treatment, compared to pretreatment scores, there were no differences on behavioral and emotional symptoms as measured by the CBCL. Finally, using a longer follow-up period of one year, Cohen and Mannarino (1997) found that reductions in sexual behavior problems were maintained. Overall, these studies provide initial indications that some treatment gains may be maintained over time; however, additional studies with similar follow-up periods are necessary to shed more light on the issue of long-term treatment effectiveness. 


\section{a}
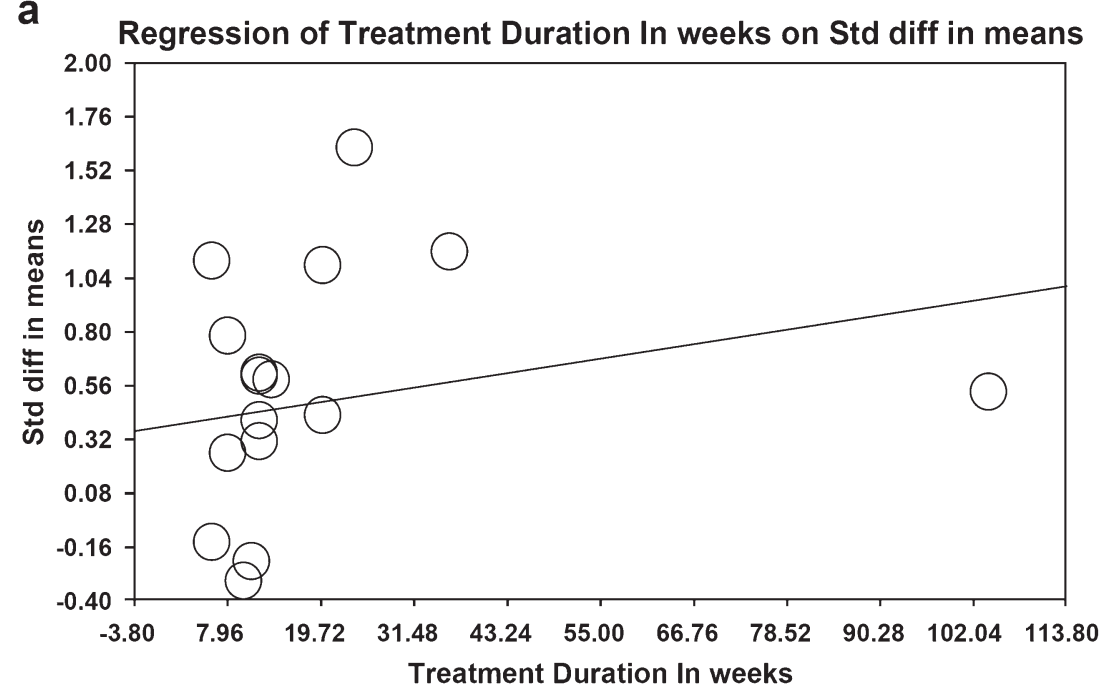

b

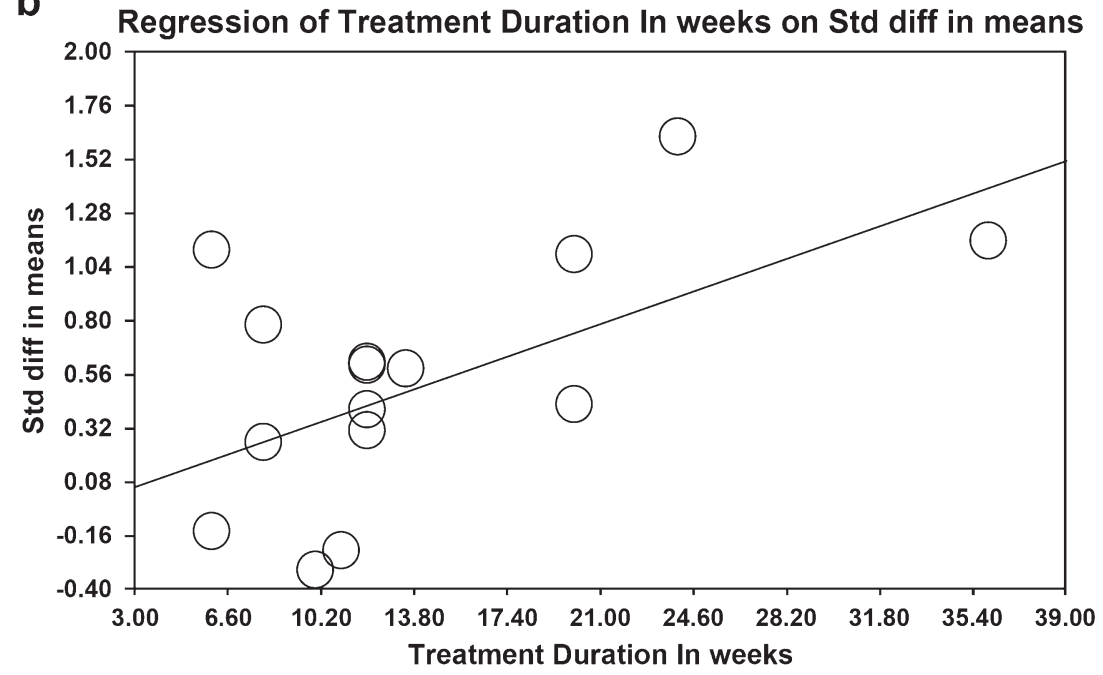

Figure 4. a. Scatter plot of treatment duration by effect sizes for studies utilizing a between-group design. $b$. Scatter plot of treatment duration by effect sizes for studies utilizing a between-group design less the Bagley article.

\section{Discussion}

The goals of this analysis were twofold: 1) to systematically evaluate the effectiveness of therapeutic interventions in reducing the most commonly documented effects of childhood sexual abuse, and; 2) to examine participant and treatment characteristics that may moderate treatment effectiveness. To accomplish these goals, 35 published studies and unpublished dissertations that utilized diverse treatment modalities, research designs, and theoretical orientations to treatment were quantitatively synthesized. In doing so we aimed to build on prior meta-analyses, which were older, left out relevant studies, or examined only one type of treatment modality, research design, or theoretical orientation to treatment.

Overall, treatment was effective in reducing many negative outcomes of CSA. The effect sizes averaged across all outcomes were medium (Cohen, 1988) for studies utilizing a single-group pretest-posttest design as well as for studies using a between-group design. These results are consistent with prior meta-analytic reviews (Hetzel-Riggin et al., 2007; Reeker et al., 1997; Skowron and Reinemann, 2005). Similar to the overall meta-analyses, therapeutic interventions also significantly decreased specific emotional problems in sexually abused children. For single-group designs, although the treatment effects were medium for PTSD symptoms, externalizing, and internalizing symptoms, the effect size for PTSD symptoms was not significant. One study that was included in that analysis (Simmer-Dvonch, 1998) had a weighted mean effect size of -.18 and was based on a sample size of 5 individuals. This study may have reduced the overall effect size for the pretestposttest studies. For between-group designs, treatment effects were medium for PTSD and internalizing problems and small for externalizing problems. The medium effect size estimates for externalizing and internalizing problems were consistent with the Reeker et al. (1997) meta-analysis, but smaller than the Hetzel-Riggin et al. (2007) meta-analysis, which reported large effect sizes using Cohen's (1988) criteria. Such disparity may be due to the different samples used by Hetzel-Riggin et al. and the current study. Specifically, Hetzel-Riggin et al. examined treatment effectiveness for outcomes associated with general child maltreatment, same-age peer rape, and CSA, whereas the current study focused on outcomes associated with CSA specifically. 
Despite these differences, the strength of these overall effect sizes provides support for the effectiveness of treatment in reducing PTSD symptoms as well as externalizing and internalizing problems experienced by child victims of CSA. In fact, the majority of effect sizes found in this study fall above the grand mean of effect sizes reported in Lipsey and Wilson's (1993) well-known examination of 302 treatment outcome meta-analyses. These meta-analyses represent a broad range of psychotherapy outcomes conducted within a variety of samples (e.g., children, adults), problem areas, and settings (e.g., school, clinic). Thus, treatment for outcomes of CSA appears to be at least as effective as psychotherapy in general, as indicated by the broader research on psychotherapy effectiveness.

\subsection{Moderators of treatment effectiveness}

\subsubsection{Study characteristics}

Our examination of publication status indicated that published studies generally had larger treatment effects than did unpublished dissertations. This finding is again consistent with Lipsey and Wilson's (1993) examination of meta-analyses, in which estimates of psychological treatment effects were larger for published than unpublished studies. Within the child area specifically, McLeod and Weisz (2004) also found stronger effects for published studies versus dissertations in their metaanalysis of the general youth psychotherapy literature. In the present analyses, both single-group and between-group designs showed this pattern. Although the between-group differences in publication status were at the trend level, our obtained effect sizes for published and unpublished studies (.59 and .36, respectively) closely resemble the standardized mean difference effect sizes reported by Lipsey and Wilson (1993; .53 and .39). Thus, our overall findings conform with prior meta-analyses and support the importance of including dissertations as a means of counteracting possible overestimation of treatment effects based only on the published literature.

Individual and group treatments were found to be equally effective regardless of study design. This finding is consistent with prior clinical writings describing the utility of group interventions for outcomes of CSA (Hansen, Hecht, \& Futa, 1998) as well as an earlier meta-analysis showing that treatment modality did not moderate effectiveness for general child maltreatment interventions, including those targeting outcomes of CSA (Skowron \& Reinemann, 2005). If individual and group interventions are indeed equally effective, this has implications for treatment development and utilization. Other factors being equal, group interventions treat several children simultaneously, and therefore are more efficient. Research suggests that clinicians and managed care companies anticipate that the use of group treatments will increase in the future (Taylor et al., 2001). Taken together, these findings indicate that group intervention for outcomes of CSA may be the most practical treatment modality for service providers to adopt.

The results of some moderator analyses differed across type of research design. For example, the inclusion of caregiver had no effect in single-group pretest-posttest analysis but was associated with lower treatment effectiveness in the betweengroup analysis. These differences may be due to the type of control group utilized by child-only treatment studies and those that also included caregivers. Specifically, six of the eight studies that examined child-only treatments utilized no-treatment comparison groups, whereas six out of the seven studies that included the caregiver utilized attention-placebo control groups. Given that no-treatment comparison groups resulted in significantly larger treatment effects than attention-placebo control groups, this may have contributed to our finding of decreased effectiveness when caregivers were included. In a similar vein, although there was no effect of treatment duration in single- group pretest-posttest studies, longer treatments were more effective in the between-group studies. This finding may reflect additional time needed in randomized trials for treatments to show effects relative to control groups. By contrast, treatment gains in single single-group studies do not depend on such comparisons and may therefore suggest quicker symptom reduction (e.g., through natural recovery or regression artifacts).

Where there were differential effects for theoretical approach, cognitive-behavioral interventions were more beneficial than treatments based on "other" theoretical models. Although these findings are based on only three single-group studies, they comport with prior meta-analyses (e.g., Macdonald et al., 2006) in suggesting that cognitive-behavioral interventions may be especially useful for reducing CSA-related symptomatology. Although the bulk of studies reviewed here $(62 \%)$ investigated treatment as usual or supportive therapy approaches, the accumulating evidence suggests that further evaluation of CBT approaches is needed. Specific cognitive-behavioral interventions, such as Trauma-Focused CBT (Cohen, Mannarino, \& Deblinger, 2006), appear especially promising and worthy of further evaluation.

\subsubsection{Participant characteristics}

Reflecting the population of youth who are sexually abused, the aggregated sample included in this meta-analysis was heterogeneous with respect to gender, and ethnicity. Child age and gender each were significant moderators in studies utilizing a between-group design. Studies with older children and those with a greater proportion of male participants revealed larger treatment effects. The finding that older children benefitted more from treatment makes intuitive sense because many existing interventions rely heavily on cognitive components (e.g., the cognitive triad, cognitive distortions), which may be easier for older children to grasp. This possibility is consistent with evidence that although younger children may benefit from cognitive therapy, these techniques must be adapted based on the age and ability of the child (Doherr, Reynolds, Wetherly, \& Evans, 2005). It is unclear to what extent studies included in this meta-analysis adjusted the cognitive components of interventions to meet children's developmental level.

The results from between-group studies that boys benefitted more from CSA treatment were unexpected and contrast with the non-significant results found in our single-group analyses and the Reeker et al. (1997) meta-analysis. It should be noted that the moderating effect found in the betweengroup meta-analysis was based on two studies with a majority of male participants; therefore, this finding warrants greater attention when more studies with male survivors of CSA become available. Ethnicity was not a factor in treatment effectiveness, regardless of study design, indicating that treatment may be equally beneficial for individuals from diverse ethnic backgrounds. These results correspond with a recent review on the effectiveness of psychosocial interventions, which reported growing evidence of the success of treatment for ethnic minority individuals (Miranda et al., 2005).

\subsection{Limitations of this meta-analysis}

Although the present study provides a needed update on the effectiveness of treatments for outcomes of CSA, certain limitations should be acknowledged. First, while every attempt was made to incorporate all published studies that met the inclusion criteria (e.g., contacting the authors, estimating effect sizes), two were excluded (Scott et al., 2003; Tourigny and Hebert, 2007) due to insufficient statistical information to calculate effect sizes. Thus, although no indication of publication bias was found, the current meta-analysis did not include all identifiable studies. Second, when a study reported 
more than one effect size representing the same outcome (e.g., PTSD), mean weighted effect sizes were calculated, which facilitated statistical independence. While this is a common approach (Gliner et al., 2003; Lipsey and Wilson, 2001), others have suggested that using the strength of the correlations between measures may be a preferable method of ensuring independence (Gliner et al.). Regrettably, this approach could not be used here because the needed information was missing from the majority of treatment outcome studies.

\subsection{Future research directions}

The present review reveals important directions for future research on the effectiveness of CSA interventions. Most notably, although randomized controlled trials are considered the gold standard for evaluating treatment outcomes (see Kazdin, 2003, Chapter 5), only 9 of 35 studies reviewed here can be classified as RCTs, suggesting that additional well controlled studies addressing this topic are necessary. This need is underscored by the somewhat different findings obtained here from single-group versus between-group studies. Although pre-experimental designs should not be discounted, these studies are likely to be most useful early in the process of treatment evaluation, as a means of revealing the potential benefits of an intervention. The most significant advances in knowledge will come from well controlled experimental studies, conducted with a diversity of client populations, using standardized treatment and assessment protocols. In addition to evaluating overall treatment effects, these studies will be strengthened by examining the participant-level moderators considered here as well as others rarely reported in the literature to date. For example, a large proportion of children who are sexually abused also have been exposed to other forms of trauma, including physical and psychological abuse, neglect, and witnessing violence (Turner, Finkelhor, \& Ormrod, 2010). Future studies should evaluate whether interventions designed for sexually abused children are also effective for children who have experienced poly-victimization, which shares many detrimental outcomes with CSA (e.g., trauma symptoms; Finkelhor, Ormrod, \& Turner, 2007). In doing so, it may also be important to examine whether the nature and degree of trauma exposure (e.g., chronicity and types of abusive acts, relationship to perpetrator) moderate treatment outcomes. Researchers reporting outcomes of such studies should provide correlations between outcome measures, which allow for more advanced analytic methods to combine effect sizes while ensuring independence. Finally, important knowledge will also be gained by including additional follow-up assessments at regular intervals (e.g., 6 months, 1 year, and 2 years) to determine whether the immediate treatment gains documented here are maintained over time. Only a handful of studies to date have examined the lasting effects of interventions, and those that did utilized varying follow-up periods. This inconsistency prevented the metaanalysis of follow-up data, which is crucial to understanding whether treatment effects are long-lasting.

Finally, although three of the most prevalent outcomes were examined here, future studies might investigate treatment effects on a wider range of CSA symptomatology, which is known to span the affective, behavioral, cognitive, and interpersonal realms of functioning. This work should evaluate the effects of interventions in addressing consequences such as emotion dysregulation and sexualized behavior, which have been frequently documented but rarely reported in the treatment outcome literature. Of course, unlike disorder-specific research, the wide symptom heterogeneity associated with CSA poses an additional obstacle for conducting treatment outcome studies. Ongoing and concerted efforts will be needed to overcome these challenges and better address the myriad needs of sexually victimized youth.
Acknowledgment - This project was supported by a National Institute of Mental Health Ruth L. Kirschstein National Research Service Award (F31 MH080533-01A1) awarded to Emily Trask, under the supervision of David DiLillo.

\section{References}

( ${ }^{*}$ References marked with an asterisk indicate studies included in the meta-analysis.)

Adams, J., McClellan, J., Douglass, D., McCurry, C., \& Storck, M. (1995). Sexually inappropriate behaviors in seriously mentally ill children and adolescents. Child Abuse \& Neglect, 19, 555-568.

*Arnold, E. M., Kirk, R. S., Roberts, A. C., Griffith, D. P., Meadows, K., \& Julian, J. (2003). Treatment of incarcerated, sexually-abused adolescent females: An outcome study. Journal of Child Sexual Abuse, 12, 123-139.

*Baker, C. R. (1985). A comparison of individual and group therapy as treatment of sexually abused adolescent females (Unpublished doctoral dissertation). University of Maryland.

*Bagley, C., \& LaChance, M. (2000). Evaluation of a family-based programme for the treatment of child sexual abuse. Child and Family Social Work, 5, 205-213.

Begg, C. B. (1994). Publication bias. In H. Cooper, \& L. V. Hedges (eds.), The handbook of research synthesis (pp. 399-409). New York: Russell Sage Foundation.

Borenstein, M., Hedges, L., Higgins, J., \& Rothstein, H. (2005). Comprehensive Metaanalysis Version 2. Englewood NJ: Biostat.

*Brown, S. (2007). An investigation of trauma symptom reduction in a clinical sample of sexually abused children using the Trauma Symptom Checklist for Children (Unpublished doctoral dissertation). Georgia State University.

*Burke, M. (1988). Short-term group therapy for sexually abused girls: A learning theory based treatment for negative affect (Unpublished doctoral dissertation). University of Georgia.

*Celano, M., Hazzard, A., Webb, C., \& McCall, C. (1996). Treatment of traumagenic beliefs among sexually abused girls and their mothers: An evaluation study. Journal of Abnormal Child Psychology, 24, 1-17.

Chaffin, M., Silovsky, J. F., \& Vaughn, C. (2005). Temporal concordance of anxiety disorders and child sexual abuse: Implications for direct versus artifactual effects of sexual abuse. Journal of Clinical Child and Adolescent Psychology, 34, 210-222.

Chen, L. P., Murad, H., Paras, M. L., Colbenson, K. M., Sattler, A. L., Goranson, E. N., et al. (2010). Sexual abuse and lifetime diagnosis of psychiatric disorders: Systematic review and meta-analysis. Mayo Clinic Proceedings, 85, 618-629.

Cohen, J. (1988). Statistical power analysis for the behavioral sciences. Hillsdale, NJ: Lawrence Erlbaum.

${ }^{*}$ Cohen, J. A., Deblinger, E., Mannarino, A. P., \& Steer, R. A. (2004). A multisite, randomized controlled trial for children with sexual abuse-related PTSD symptoms. Journal of the American Academy of Child and Adolescent Psychiatry, 43, 393-402.

Cohen, J. A., Mannarino, A. P., \& Deblinger, E. (2006). Treating trauma and traumatic grief in children and adolescents. New York: The Guilford Press.

*Cohen, J. A., \& Mannarino, A. P. (1997). A treatment outcome study for sexually abused preschool children: Outcome during one year follow-up. Journal of the American Academy of Child and Adolescent Psychiatry, 36, 1228-1235.

*Cohen, J. A., \& Mannarino, A. P. (1998). Interventions for sexually abused children: Initial treatment outcome findings. Child Maltreatment, 3, 17-26.

*Costas, M. B. (1998). Filial therapy with non-offending parents of children who have been sexually abused (Unpublished doctoral dissertation). University of North Texas.

*Deblinger, E., Lippmann, J., \& Steer, R. (1996). Sexually abused children suffering posttraumatic stress symptoms: Initial treatment outcome findings. Child Maltreatment, 1, 310-322.

*Deblinger, E., McLeer, S. V., \& Henry, D. (1990). Cognitive behavioral treatment for sexually abused children suffering post-traumatic 
stress: Preliminary findings. Journal of the American Academy of Child and Adolescent Psychiatry, 29, 747-752.

*Deblinger, E., Stauffer, L. B., \& Steer, R. A. (2001). Comparative efficacies of supportive and cognitive behavioral group therapies for young children who have been sexually abused and their nonoffending mothers. Child Maltreatment, 6, 332-343.

*De Luca, R. V., \& Hazen, A. (1993). Evaluation of a group counseling program for preadolescent female victims of incest. Elementary School Guidance \& Counseling, 28, 104-114.

Doherr, L., Reynolds, S., Wetherly, J., \& Evans, E. H. (2005). Young children's ability to engage in cognitive therapy tasks: Associations with age and educational experience. Behavioural and Cognitive Psychotherapy, 33, 201-215.

Dubner, A. E., \& Motta, R. W. (1999). Sexually and physically abused foster care children and posttraumatic stress disorder. Journal of Consulting and Clinical Psychology, 67, 367-373.

Dubowitz, H., Black, M., Harrington, D., \& Verschoore, A. (1993). A follow-up study of behavior problems associated with child sexual abuse. Child Abuse \& Neglect, 17, 743-754.

*Duffany, A., \& Panos, P. T. (2009). Outcome evaluation of a group treatment of sexually abused and reactive children. Research on Social Work Practice, 19, 291-303.

Fergusson, D. M., Boden, J. M., \& Horwood, L. J. (2008). Exposure to childhood sexual and physical abuse and adjustment in early adulthood. Child Abuse \& Neglect, 32, 607-619.

Finkelhor, D., \& Berliner, L. (1995). Research on the treatment of sexually abused children: A review and recommendations. Journal of the American Academy of Child and Adolescent Psychiatry, 34, 1408-1423.

Finkelhor, D., Ormrod, R. K., \& Turner, H. A. (2007). Poly-victimization: A neglected component in child victimization. Child Abuse \& Neglect, 31, 7-26.

*Friedrich, W. N., Luecke, W. J., Beilke, R. L., \& Place, V. (1992). Psychotherapy outcome of sexually abused boys: An agency study. Journal of Interpersonal Violence, 7, 396-409.

Gliner, J. A., Morgan, G. A., \& Harmon, R. J. (2003). Meta-analysis: Formulation and interpretation. Journal of the American Academy of Child and Adolescent Psychiatry, 42, 1376-1379.

Gray, A., Pithers, W. D., Busconi, A., \& Houchens, P. (1999). Children with sexual behavior problems and their caregivers: Demographics, functioning, and clinical patterns. Sexual Abuse: A Journal of Research and Treatment, 9, 267-290.

*Hack, T. F., Osachuk, T. A., \& De Luca, R. V. (1994). Group treatment for sexually abused preadolescent boys. Services, 75, 217-228.

*Hall-Marley, S. E., \& Damon, L. (1993). Impact of structured group therapy on young victims of sexual abuse. Journal of Child and Adolescent Group Therapy, 3, 41-48.

Hansen, D. J., Hecht, D. B., \& Futa, K. T. (1998). Child sexual abuse. In V. B. Van Hasselt, \& M. Hersen (Eds.), Handbook of psychological treatment protocols for children and adolescents (pp. 153-178). New York: Lawrence Erlbaum.

Hansen, D. J., Warner-Rogers, J. E., \& Hecht, D. B. (1998). Implementing and evaluating an individualized behavioral intervention program for maltreating families: Clinical and research issues. In J. R. Lutzker (Ed.), Handbook of child abuse research and treatment (pp. 133-158). New York: Plenum Press.

Hetzel-Riggin, M. D., Brausch, A. M., \& Montgomery, B. S. (2007). A meta-analytic investigation of therapy modality outcomes for sexually abused children and adolescents: An exploratory study. Child Abuse \& Neglect, 31, 125-141.

*Hiebert-Murphy, D., De Luca, R. V., \& Runtz, M. (1992). Group treatment for sexually abused girls: Evaluating outcomes. Families in Society: The Journal of Contemporary Human Services, 73, 205-213.

Higgins, J., \& Green, S. (Eds.). (2009). Cochrane Handbook for Systematic Reviews of Interventions 5.0.2 [updated September, 2009]. www.cochrane-handbook.org (accessed February, 10th, 2010).

Hoagwood, K., Hibbs, E., Brent, D., \& Jensen, P. (1995). Introduction to the special section: Efficacy and effectiveness in studies of child and adolescent psychotherapy. Journal of Consulting and Clinical Psychology, 63, 683-687.
*Hsu, E. (2003). Parallel group treatment for sexually abused children and their nonoffending parents: An examination of treatment integrity and child and family outcome and satisfaction (Unpublished doctoral dissertation). University of Nebraska-Lincoln.

*Humberson, M. A. (1998). A preliminary investigation of the impact of a parallel group model of treatment on behavior problems in young victims of sexual abuse (Unpublished doctoral dissertation). California School of Professional Psychology, Los Angeles.

*Hyde, C., Bentovim, A., \& Monck, E. (1995). Some clinical and methodological implications of a treatment outcome study of sexually abused children. Child Abuse \& Neglect, 19, 1387-1399.

Jaberghaderi, N., Greenwald, R., Rubin, A., Zand, S. O., \& Dolatabadi, S. (2004). A comparison of CBT and EMDR for sexually-abused Iranian girls. Clinical Psychology \& Psychotherapy, 11, 358-368.

Jones, L. M., Finkelhor, D., \& Halter, S. (2006). Child maltreatment trends in the 1990s: Why does neglect differ from sexual and physical abuse? Child Maltreatment, 11, 107-120.

Kazdin, A. E. (2003). Research design in clinical psychology. Boston, MA: Allyn \& Bacon.

Kazdin, A. E., \& Nock, M. K. (2003). Delineating mechanisms of change in child and adolescent therapy: Methodological issues and research recommendations. Journal of Child Psychology and Psychiatry, 44, 1116-1129.

Kendall-Tackett, K., Williams, L., \& Finkelhor, D. (1993). Impact of sexual abuse on children: A review and synthesis of recent empirical studies. Psychological Bulletin, 113, 164-180.

Kendall-Tackett, K. A., Williams, L. M., \& Finkelhor, D. (2001). Impact of sexual abuse on children: A review and synthesis of recent empirical studies. In R. Bull, \& R. E. Bull (Eds.), Children and the law: The essential readings (pp. 31-76). Malden: Blackwell Publishing.

*King, N. J., Tonge, B., Mullen, P., Myerson, N., Heyne, D., Rollings, S., et al. (2000). Treating sexually abused children with posttraumatic stress symptoms: A randomized clinical trial. Journal of the American Academy of Child and Adolescent Psychiatry, 39, 1347-1355.

Koverola, C., Pound, J., Heger, A., \& Lytle, C. (1993). Relationship of child sexual abuse to depression. Child Abuse \& Neglect, 17, 393-400.

Kraemer, H. C., Wilson, C. G., Fairburn, T., \& Agras, S. (2002). Mediators and moderators of treatment effects in randomized clinical trials. Archives of General Psychiatry, 59, 877-883.

Krakow, B., Sandoval, D., Schrader, R., Keuhne, B., Yau, C. L., \& Tandberg, D. (2001). Treatment of chronic nightmares in adjudicated adolescent girls in a residential facility. The Journal of Adolescent Health, 29, 94-100.

*Lanktree, C. B., \& Briere, J. (1995). Outcome of therapy for sexually abused children: A repeated measures study. Child Abuse \& Neglect, 19, 1145-1155.

Lipsey, M. W. (1992). Juvenile delinquency treatment: A meta-analytic inquiry into variability of effects. In T. D. Cook, H. Cooper, D. S. Cordray, H. Hartmann, L. V. Hedges, R. L. Light, \& T. A. Louis (Eds.), Meta-analysis for explanation (pp. 83-127). New York: Russell-Sage Foundation.

Lipsey, M. W., \& Wilson, D. B. (1993). The efficacy of psychological, educational, and behavioral treatment: Confirmation from metaanalysis. The American Psychologist, 48, 1181-1209.

Lipsey, M. W., \& Wilson, D. B. (2001). Practical meta-analysis, Vol. 49, Thousand Oaks, CA: Sage Publications.

Lynskey, M. T., \& Fergusson, D. M. (1997). Factors protecting against the development of adjustment difficulties in young adults exposed to childhood sexual abuse. Child Abuse \& Neglect, 21, 1177-1190.

Macdonald, G. M., Higgins, J. P. T., \& Ramchandani, P. (2006). Cognitive-behavioural interventions for children who have been sexually abused. Cochrane Database of Systematic Reviews 2006, 4. Art. No. CD001930.

*MacKay, B., Gold, M., \& Gold, E. (1987). A pilot study in drama therapy with adolescent girls who have been sexually abused. The Arts in Psychotherapy, 14, 77-84.

*McGain, B., \& McKinzey, R. K. (1995). The efficacy of group treatment in sexually abused girls. Child Abuse \& Neglect, 19, 1157-1169. 
McLeer, S. V., Deblinger, E., Atkins, M. S., Foa, E. B., \& Ralphe, D. L. (1988). Post-traumatic stress disorder in sexually abused children. Journal of the American Academy of Child and Adolescent Psychiatry, 27, 650-654.

McLeer, S. V., Deblinger, E., Henry, D., \& Orvaschel, H. (1992). Sexually abused children at high risk for PTSD. Journal of the American Academy of Child and Adolescent Psychiatry, 31, 875-879.

McLeer, S. V., Dixon, J. F., Henry, D., Ruggiero, K., Escovitz, K., Niedda, T., et al. (1998). Psychopathology in non-clinically referred sexually abused children. Journal of the American Academy of Child and Adolescent Psychiatry, 37, 1326-1333.

McLeod, B. D., \& Weisz, J. R. (2004). Using dissertations to examine potential bias in child and adolescent clinical trials. Journal of Consulting and Clinical Psychology, 72, 235-251.

Miranda, J., Bernal, G., Lau, A., Kohn, L., Hwang, W., \& LaFromboise, T. (2005). State of the science on psychosocial interventions for ethnic minorities. Annual Review of Clinical Psychology, 1, 113-142.

${ }^{*}$ Monck, E. (1997). Evaluating therapeutic intervention with sexually abused children. Child Abuse Review, 6, 163-177.

Morris, S. B., \& DeShon, R. P. (2002). Combining effect size estimates in meta-analysis with repeated measures and independent-groups designs. Psychological Methods, 7, 105-125.

Nalavany, B. A., Ryan, S. D., \& Hinterlong, J. (2009). Externalizing behavior among adopted boys with preadoptive histories of child sexual abuse. Journal of Child Sexual Abuse: Research, Treatment, \& Program Innovations for Victims, Survivors, \& Offenders, 18, 553-573.

Neumann, D. A., Houskamp, B. M., Pollock, V. E., \& Briere, J. (1996). The long-term sequelae of childhood sexual abuse in women: A meta-analytic review. Child Maltreatment, 1, 6-16.

Noll, J. G. (2008). Sexual abuse of children-Unique in its effects on development? Child Abuse \& Neglect, 32, 603-605.

Pereda, N., Guilera, G., Forns, M., \& Gomez-Benito, J. (2009). The prevalence of child sexual abuse in community and student samples: A meta-analysis. Clinical Psychology Review, 29, 328-338.

Putnam, F. W. (2003). Ten-year research update review: Child sexual abuse. Journal of the American Academy of Child and Adolescent Psychiatry, 42, 269-278.

*Reeker, J., \& Ensing, D. (1998). An evaluation of a group treatment for sexually abused young children. Journal of Child Sexual Abuse, 7, 65-85.

Reeker, J., Ensing, D., \& Elliott, R. (1997). A meta-analytic investigation of group treatment outcomes for sexually abused children. Child Abuse \& Neglect, 21, 669-680.

Romano, E., \& De Luca, R. V. (2001). Male sexual abuse: A review of effects, abuse characteristics, and links with later psychological functioning. Aggression and Violent Behavior, 6, 55-78.

Romano, E., Zoccolillo, M., \& Paquette, D. (2006). Histories of child maltreatment and psychiatric disorder in pregnant adolescents. Journal of the American Academy of Child and Adolescent Psychiatry, 45, 329-336.

Sawyer, G. K. (2007). Heterogeneous Symptoms Patterns of Sexually Abused Youth in Treatment (Unpublished doctoral dissertation). University of Nebraska-Lincoln.

Saywitz, K. J., Mannarino, A. P., Berliner, L., \& Cohen, J. A. (2000). Treatment of sexually abused children and adolescents. The American Psychologist, 55, 1040-1049.

Scott, T. A., Burlingame, G., Starling, M., Porter, C., \& Lilly, J. P. (2003). Effects of individual client-centered play therapy on sexually abused children's mood, self-concept, and social competence. International Journal of Play Therapy, 12, 7-30.

Sheu, C., \& Suzuki, S. (2001). Meta-analysis using linear mixed models. Behavior Research Methods, Instruments, \& Computers, 33, 102-107.

*Silveria, D. D. (1994). Cognitive-behavioral group therapy for sexually abused adolescent males (Unpublished doctoral dissertation). University of Southern California.
Silverman, W. K., Ortiz, C. D., Viswesvaran, C., Burns, B. J., Kolko, D. J., Putnam, F. W., et al. (2008). Evidence-based psychosocial treatments for children and adolescents exposed to traumatic events. Clinical Child and Adolescent Psychology, 37, 156-183. *SimmerDvonch, L. M. (1999). Development and evaluation of a group treatment program for sexually abused male adolescents (Unpublished doctoral dissertation). The University of Toledo, Canada.

*Sinclair, J. J., Larzelere, R. E., Paine, M., Jones, P., Graham, K., \& Jones, M. (1995). Outcome of group treatment for sexually abused adolescent females living in a group home setting. Journal of Interpersonal Violence, 10, 533-542.

Skowron, E., \& Reinemann, D. (2005). Effectiveness of psychological interventions for child maltreatment: A meta-analysis. Psychotherapy: Theory, Research, Practice, Training, 4, 52-71.

Spataro, J., Mullen, P. E., Burgess, P. M., Wells, D. L., \& Moss, S. A. (2004). Impact of child sexual abuse on mental health: Prospective study in males and females. The British Journal of Psychiatry, 184, 416-421.

*Stauffer, L. B., \& Deblinger, E. (1996). Cognitive behavioral groups for nonoffending mothers and their young sexually abused children: A preliminary treatment outcome study. Child Maltreatment, 1, 65-76.

Stevenson, J. (1999). The treatment of the long-term sequelae of child abuse. Journal of Child Psychology and Psychiatry, 40, 89-111.

*Sullivan, P. M., Scanlan, J. M., Brookhouser, P. E., Schulte, L. E., \& Knutson, J. F. (1992). The effects of psychotherapy on behavior problems of sexually abused deaf children. Child Abuse \& Neglect, 16, 297-307.

Swanston, H. Y., Parkinson, P. N., O'Toole, B. I., Plunkett, A. M., Shrimpton, S., \& Oates, R. K. (2003). Juvenile crime, aggression and delinquency after sexual abuse: A longitudinal study. British Journal of Criminology, 43, 729-749.

Target, M., \& Fonagy, P. (1996). The psychological treatment of child and adolescent psychiatric disorders. In A. Roth, \& P. Fonagy (Eds.), What works for whom? A critical review of psychotherapy research (pp. 263-320). New York: Guilford Press.

Taylor, N. T., Burlingame, G. M., Kristensen, K. B., Fuhriman, A., Johansen, J., \& Dahl, D. (2001). A survey of mental health care provider's and managed care organization attitudes toward familiarity with, and use of group interventions. International Journal of Group Psychotherapy, 51, 243-263.

Tebbutt, J., Swanston, H., Oates, R. K., \& O'Toole, B. I. (1997). Five years after child sexual abuse: Persisting dysfunction and problems of prediction. Journal of the American Academy of Child and Adolescent Psychiatry, 36, 330-339.

Tourigny, M., \& Hebert, M. (2007). Comparison of open versus closed group interventions for sexually abused adolescent girls. Violence and Victims, 22, 334-348.

*Tourigny, M., Hebert, M., Daigneault, I., \& Simoneau, A. C. (2005). Efficacy of a group therapy for sexually abused adolescent girls. Journal of Child Sexual Abuse, 14, 71-89.

Tremblay, C., Hebert, M., \& Piche, C. (2000). Type I and type II posttraumatic stress disorder in sexually abused children. Journal of Child Sexual Abuse, 9, 65-90.

Trickett, P. K., \& McBride-Chang, C. (1995). The developmental impact of different forms of child abuse and neglect. Developmental Review, 15, 311-337.

Turner, H. A., Finkelhor, D., \& Ormrod, R. (2010). Poly-victimization in a national sample of children and youth. American Journal of Preventive Medicine, 38, 323-330.

Weinstein, D., Staffelbach, D., \& Biaggio, M. (2000). Attention-deficit hyperactivity disorder and posttraumatic stress disorder: Differential diagnosis in childhood sexual abuse. Clinical Psychology Review, 20, 359-378. 